\title{
PHENOTYPIC VARIABILITY IN A MEXICAN MESTIZO FAMILY WITH RETINAL VASCULOPATHY WITH CEREBRAL LEUKODYSTROPHY AND TREX1 MUTATION P.V235GFS*6
}

\author{
Nancy Monroy-Jaramillo ${ }^{1}$, Aurelio Cerón ${ }^{2}$, Elizabeth León ${ }^{2}$, Verónica Rivas 3 , Adriana \\ Ochoa-Morales ${ }^{1}$, María Georgina Arteaga-Alcaraz ${ }^{4}$, Fausto Carlos Nocedal-Rustrian ${ }^{5}$, \\ Cecilia Gallegos ${ }^{6}$, María Elisa Alonso-Vilatela ${ }^{1}$, Teresa Corona $3^{\dagger}$, José Flores ${ }^{3 *}$
}

${ }^{1}$ Department of Neurogenetics, ${ }^{2}$ Department of Neurology and ${ }^{3}$ Clinical Laboratory of Neurodegenerative Diseases, Instituto Nacional de Neurología y Neurocirugía Manuel Velasco Suárez, Mexico City; ${ }^{4}$ State Research Coordination, Mexican Social Security Institute (IMSS), Pachuca, Hgo.; ${ }^{5}$ Hospital Regional \# 25 Ignacio Zaragoza, Mexican Social Security Institute, Mexico City; ${ }^{6}$ Department of Pathology, Hospital Ángeles del Pedregal, Mexico City, Mexico

\begin{abstract}
Background: Retinal vasculopathy with cerebral leukodystrophy (RVCL) is an adult-onset, autosomal dominant disease involving microvessels of the brain and eye resulting in central nervous system degeneration with visual disturbances, stroke, motor impairment, and cognitive decline. Frameshift mutations at the C-terminus of TREX1 gene are the molecular cause of this disorder. Objectives: The objective of this study is to present the different clinical manifestations of RVCL in three-related patients and to investigate the presence of TREX1 mutation in the extended genealogy. Methods: Multidisciplinary testing was performed in three related patients. Based on their family history, the study was extended to 34 relatives from the same small community. Neurological evaluation, sequencing of TREX1, and presymptomatic diagnosis were offered to all participants. Results: The patients exhibited the heterozygous TREX1 mutation p.V235Gfs*6, but with phenotypic variability. In addition, 15 relatives were identified as pre-manifest mutation carriers. The remaining participants did not carry the mutation. Conclusions: This is the first report of a large Mexican genealogy with RVCL, where the same TREX1 mutation causes a variation in organ involvement and clinical progression. The early identification and follow-up of individuals at risk may help provide insights into the basis for this variability in presentation. (REV INVES CLIN. 2018;70:68-75)
\end{abstract}

Key words: Retinal vasculopathy with cerebral leukodystrophy. Phenotypic variability. pseudotumoral demyelinating lesion. TREX1 gene. Presymptomatic diagnosis.

Corresponding author:

*José Flores

Instituto Nacional de Neurología y Neurocirugía

Insurgentes Sur, 3877

Col. La Fama, Del. Tlalpan

C.P. 14269, Mexico City, Mexico

E-mail: nancy97@unam.mx
†These authors contributed equally to this work.

Received for publication: 22-01-2018

Accepted for publication: 07-03-2018

doi: 10.24875/RIC.18002492 


\section{INTRODUCTION}

The clinical entities cerebroretinal vasculopathy (CRV); hereditary endotheliopathy with retinopathy, neuropathy, and stroke; hereditary vascular retinopathy; and hereditary systemic angiopathy have been demonstrated to be linked to the same locus and are combined as RV with cerebral leukodystrophy (RVCL) (MIM\#192315). RVCL is a devastating autosomaldominant condition whose symptoms generally appear in young adulthood, so RVCL can be passed to the next generation before a diagnosis is made. There have been 12 families known to be affected with RVCL worldwide ${ }^{1-3}$.

Clinically, RVCL is characterized by microvascular endotheliopathy leading to central nervous system (CNS) degeneration and retinopathy. Diseased cerebral white matter exhibits prominent, small infarcts that often coalesce into pseudotumors. Death can occur within 10 years of onset of symptoms ${ }^{1}$.

RVCL is associated with mutations on the TREX1 gene (3p21.31) (MIM\#606609). To date, 66 mutations have been published (HGMDB), and from these only five variants have been documented to cause RVCL. In addition, different TREX1 mutations have been associated with Aicardi-Goutières syndrome, familial chilblain lupus, and systemic lupus erythematosus ${ }^{2-5}$. TREX1 mutations associated to RVCL can lead to its altered intracellular localization or insufficient production of enzymes in the correct location to fulfill its physiological role ${ }^{2-5}$.

TREX1 encodes a ubiquitous exonuclease whose Cterminal region is located in the cytosolic compartment but during S-phase and in response to genotoxic stress, may relocate to the cell nucleus ${ }^{6,7}$.

Here, we describe the first large Mexican-Mestizo genealogy with RVCL. Clinical details are given on three-related patients with previous misdiagnoses and variable expressivity. Molecular testing identified a mutation in TREX1 indicating an unequivocal RVCL diagnosis. The study was extended to 34 relatives, all of whom originated from the same small community.

\section{SUBJECTS AND METHODS}

\section{Case 1 (index case)}

A 52-year-old male with a clinical history of kidney stones, chronic orchitis, hypertension, and progressive visual impairment was admitted in our institution. His father had systemic hypertension and his paternal grandmother multiple sclerosis, but confirmation is no longer possible. 2 years before admission, the patient was evaluated by an ophthalmologist due to acute bilateral visual loss and diagnosed with bilateral optic neuritis based on the clinical findings, without radiographic confirmation. The patient was treated with retrobulbar steroid injections during 3 days showing partial recovery of visual acuity for approximately 1 month. However, in the following months, the patient referred loss of visual acuity, evidenced by the lower capacity for reading and difficulty in distinguishing the outlines of letters. The patient presented himself at the emergency room with at least 5 days of progressive gait impairment, decreased strength in the right hand, and numbness in the right leg. At that moment, the neurological examination revealed right hemicorporal pyramidal disorder, and the patient was admitted to the demyelinating diseases' outpatient clinic in our institution. The ophthalmological findings were reduced visual acuity of counting fingers at five feet in the right eye, and 20/60 in the left eye, bilateral dyschromatopsia ( $0 / 8$ and $3 / 8$ for right and left eye, respectively). The visual field findings revealed a central and temporal island of vision in the right eye and a superonasal defect in the left eye. The funduscopic examination revealed Grade 1 vitreous hemorrhage in the right eye with optic disc pallor and sheathing of retinal vessels in both eyes (not shown).

Laboratory testing (Table 1) showed renal impairment, mild transaminases elevation, cerebrospinal fluid with an increase in proteins and lactate. Neurological examination revealed right hemiparesis (muscle strength scored as $3 / 5$, and muscle stretch reflexes scored +++$)$. The head magnetic resonance imaging (MRI) exhibited a pseudotumoral demyelinating lesion (Fig. $1 \mathrm{~A}$ and $\mathrm{B}$ ), and this was the diagnosis considered before the molecular study. Smoking and drinking habits were interrogated since they are considered risk factors for vascular diseases. Case 1 did not have a smoking habit and initiated alcohol consumption at 17 years of age. The patient received 
Table 1. Demographic data, clinical, laboratory and brain MRI findings, and medical treatments in the three patientswith RVCL

\begin{tabular}{|c|c|c|c|}
\hline Data and findings & Case 1 & Case 2 & Case \\
\hline \multicolumn{4}{|l|}{ Demographic data } \\
\hline Smoke & - & $x$ & $x^{e}$ \\
\hline Alcohol intake & $x$ & $x$ & $x$ \\
\hline Cocaine exposure & - & - & (-) \\
\hline Hypertension & $x$ & - & $x$ \\
\hline \multicolumn{4}{|l|}{ Clinical findings } \\
\hline Retinopathy & $x$ & $-{ }^{a}$ & $x$ \\
\hline Pyramidal findings & $x$ & $x$ & $x$ \\
\hline Nephropathy ${ }^{b}$ & $x$ & - & - \\
\hline Migraine & - & $x$ & $\mp$ \\
\hline Ischemic heart disease & - & - & $x$ \\
\hline \multicolumn{4}{|l|}{ Laboratory findings } \\
\hline Anti-DNA autoantibodies & $x$ & - & $=$ \\
\hline TGO and TGP more than two times elevation & $x$ & $x$ & $x$ \\
\hline Serum beta-2 microglobulin & - & - & $x$ \\
\hline Renal impairment & $x$ & - & - \\
\hline \multicolumn{4}{|l|}{ Brain MRI findings } \\
\hline Periventricular hyperintensities and pseudotumoral lesion & $x$ & $x$ & $x$ \\
\hline Treatment & & & $\frac{1}{2}$ \\
\hline Rituximab & $x$ & & $\bar{x}$ \\
\hline Intravenous steroids ${ }^{\mathrm{c}}$ & $x$ & $x$ & 0 \\
\hline Intravenous immunoglobulins ${ }^{d}$ & & & $\bar{x}$ \\
\hline Cyclophosphamide & $x$ & & $x$ \\
\hline Azathioprine & & $x$ & . \\
\hline
\end{tabular}

$\mathrm{X}$ symbol denotes the laboratory finding is present or is positive in the patient.

${ }^{a}$ Case 2 did not show any relevant eye findings at the clinical exploration, but this was done out of our institution.

bData evidenced by serum creatinine elevations more than $2.0 \mathrm{mg} / \mathrm{dL}$.

' $1 \mathrm{~g}$ intravenous in acute stage.

${ }^{\mathrm{d}} 0.4 \mathrm{mg} / \mathrm{kg} / \mathrm{d}$ by five consecutive days.

eThis patient stopped smoking 10 years ago

MRI: Magnetic resonance imaging; RVCL: Retinal vasculopathy with cerebral leukodystrophy.

methylprednisolone for 5 days without clinical improvement and was then started on a rituximab treatment. The patient has been clinically stable during follow-up.

\section{Case 2}

A 46-year-old female patient, the first-cousin of cases 1 and 3, was referred to our institution. Patient's family history of importance includes a sister deceased from high-grade glioma; father deceased due to an unspecified brain tumor, and two siblings with a history of a cerebrovascular ischemic event.

Her relevant clinical history was recurrent episodes of pulsatile holocranial headache (twice a month), sometimes accompanied by nausea and vomit, and treated with nonsteroidal anti-inflammatory drugs. A general physician diagnosed her with a migraine without aura. 3 years later, she experienced right pyramidal signs, sudden decrease in strength on the right side of the body, which determined limitation for ambulation. The patient was diagnosed with a cerebrovascular ischemic event at another institution, received only supportive therapy, and neurological rehabilitation. Over the following months, she showed some gait improvement, being able to walk with a cane and recovered partial functionality of the right upper extremity. 1 year after, the event described a new clinical event with aphasia and progressive right hemiparesis was documented (Fig. 1 C and D). A brain biopsy was performed outside of our institution 
Figure 1. Magnetic resonance imaging (MRI) radiological findings in Case 1. (A) FLAIR hyperintensities and pseudotumoral left periventricular lesion. (B) Gadolinium ring enhancement in the left periventricular lesion. MRI radiological findings in Case 2. (C) T2 hyperintensities, right frontal periventricular demyelinating lesion (arrowed) and left posterior periventricular pseudotumoral lesion. (D) Gadolinium ringenhancing lesion (arrowed).
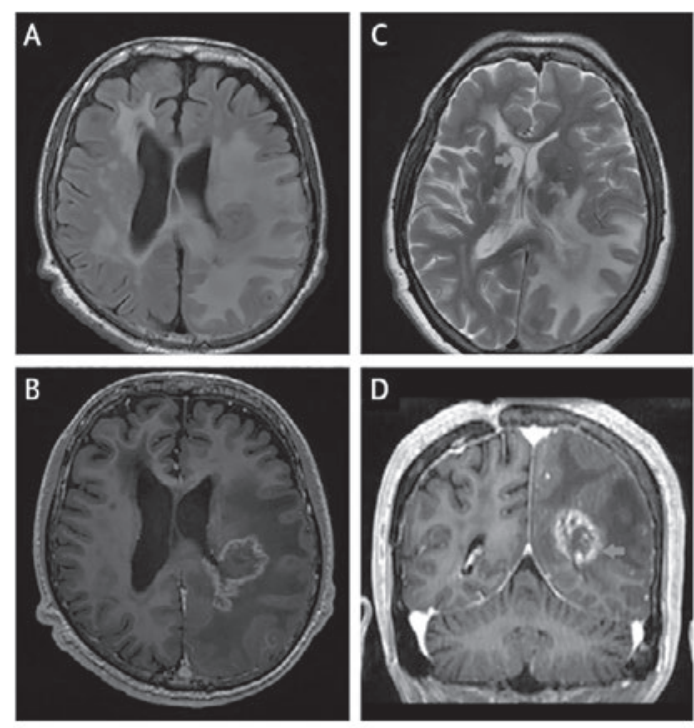

reporting vasculitis. The patient was discharged and treated with oral steroids for 3 years. 4 years later, she had a similar episode. A brain MRI (Fig. 1C and D) and a new biopsy were performed, reporting fibrinoid thrombosis (Fig. S1). Patient 2 smoked 10 cigarettes per day and reported occasional alcohol consumption.

The patient was treated with immunosuppressors (azathioprine PO, $3.0 \mathrm{mg} / \mathrm{kg} /$ day) (Table 1 ). Her last visit to the outpatient clinic was 16 months after the clinical event, without showing clinical improvement; furthermore, pyramidal findings were augmented, with increased spasticity in her lower limbs. Her family reported the patient passed away at the end of last year.

\section{Case 3}

A 50-year-old male, brother of case 1 , was previously misdiagnosed with multiple sclerosis. He reported clinical history of ischemic heart disease manifested as acute myocardial infarction at the age of 40; 7 years later, a sudden decrease in visual acuity in both eyes was added, and after ophthalmologic evaluation with fundoscopy, he was diagnosed with idiopathic bilateral retinal vasculitis and received antivascular endothelial growth factor as treatment, with partial recovery of vision. 3 years later, the patient was admitted to our institution, and a neurological examination revealed right hemiparesis (muscle strength scored as 2/5 in the upper limb, 1/5 in the lower limb, with Babinski and muscle stretch reflexes scoring +++ ) limiting motion and daily activities for the right upper limb. The neuro-ophthalmological evaluation showed bilateral intravitreal bleeding (Table 1). Brain MRI and biopsy were performed, indicating a pseudotumoral demyelinating lesion (data not available). The patient consumed 5-6 cigarettes per day, but he stopped smoking more than 10 years ago. $\mathrm{He}$ is a former drinker.

The patient was treated with intravenous immunoglobulins, with clinical improvement, and was discharged 1 month later. Since then, he has been administered intravenous cyclophosphamide (CPM) monthly. His last visit to the outpatient clinic was 24 months after the event, and he was found clinically stable.

All the patients from this study came from a village located in the State of Mexico, and all indicated that there were additional affected relatives over several generations.

Owing to clinical suspicion of RVCL (Table 1 ), we searched for TREX1 mutations in the proband (Fig. 2). Molecular testing confirmed the diagnosis of RVCL. Since all the patients included in this study belonged to the same family and because of the mode of inheritance of RVCL, we extended the study to at-risk relatives who wanted to participate in the protocol by completing a home visit voluntarily.

Thirty-four samples from related subjects (Fig. 2) were collected. Two independent neurologists evaluated all participants, and a well-structured clinical interview was conducted asking directly about any neurological symptoms. Participants signed informed consents, and the protocol was approved by local research and ethical committees.

A pedigree was constructed with information obtained from several interviews with various family members and with the help of a local historian (Fig. 2). Peripheral blood DNA was extracted, and sequencing of the 
Figure 2. Mexican genealogy with retinal vasculopathy with cerebral leukodystrophy. Molecular analysis of the TREX1 gene revealed the carrier status of the relatives that were studied. Black-filled symbols represent affected individuals; white left halffilled symbols denote pre-manifest gene mutation carriers. Individuals with a negative result for TREX1 mutation are shown with upper half-filled symbols. Double lines denote consanguinity. A boxed family indicates consanguinity in a marriage of mutation carriers.

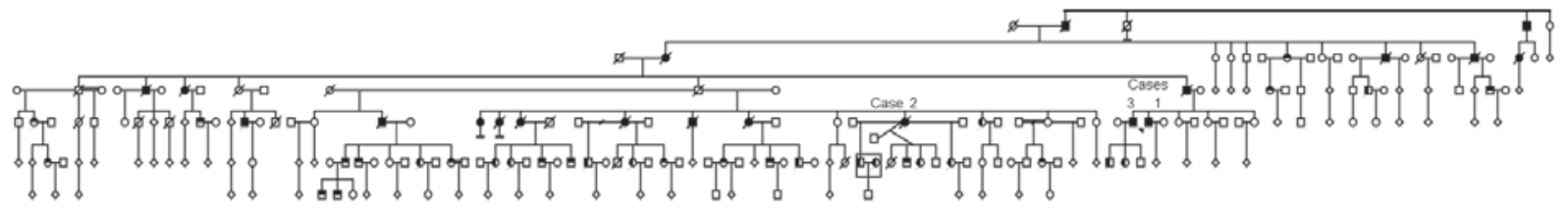

only exon of TREX1 was carried out in all participants using conditions kindly provided by Richards et al. ${ }^{1}$ The sequences were analyzed using the AB3130 (Applied Biosystems, Carlsbad, CA, USA) system and the GenBank NM_033629 file as reference sequence.

After the request of several participants who wanted to know their molecular testing results and based on experiences with genetic counseling and predictive testing of Huntington's disease in the Department of Genetics at our institution, presymptomatic diagnosis (PD) was offered to at-risk persons. Following international guidelines proposed by the World Federation of Neurology ${ }^{8}$ and specific guidelines for PD ${ }^{9}, 11$ individuals applied for PD after signing an informed consent form.

\section{RESULTS}

Both the proband and his brother (Cases 1 and 3) possessed the previously reported heterozygous c.703_704insG insertion in TREX $1^{1}$. According to the translate tool from ExPASy, this frameshift mutation (p.V235Gfs*6) truncates the resultant protein (Fig. S2). After confirming segregation of the mutation in both siblings, sequencing of TREX 1 was undertaken for the available individuals. The insertion was identified in case 2 and 15 additional relatives (Fig. 2), all of whom were healthy at the time of physical examination, and negative for any symptom of RVCL as documented in the clinical interview. For these last cases, considered as pre-manifest TREX1 gene mutation carriers, an MRI study was not deemed required since the interview was well structured and covered every aspect of the RVCL diagnosis. The remaining 19 participants did not carry the mutation.
Our three patients demonstrate intrafamilial phenotypic variability in RVCL including the age of presentation, severity of symptoms, organ involvement (Table 1 ), and clinical course of the illness.

Eleven relatives were admitted for PD, including 6 men and 5 , women with a mean age of 28 years (SD 6.8, $\mathrm{R}$ 20-40). The average years of schooling were 14.3 years (SD 2.9, R 9-17). After neuropsychological testing and psychiatric interviews, only eight people were given the results: 6 individuals were positive for the mutation, and two were negative. The remaining three individuals, who had symptoms of depression and anxiety, were judged to be unfit to receive their molecular results and were referred for psychiatric attention. Once the psychiatrist considers that they can reapply for the study, the outcome will be provided. All participants on the PD program were offered to have a file opened at our Institute for monitoring and attention purposes.

\section{DISCUSSION}

This is the first report of a Mexican family presenting RVCL and one of the largest genealogies carrying a TREX1 mutation that has been documented to date.

In this work, we have highlighted the intrafamilial phenotypic variability in three cases with $\mathrm{RVCL}$, which in turn leads to a delayed diagnosis. The family history and the multidisciplinary approach (including neurologists, radiologists, ophthalmologists, internists, and geneticists) narrowed the diagnosis to RVCL, and the genetic analysis confirmed a previously described TREX1 mutation1 (variant: Cl075707 in HGMDB) in the three clinical cases. The c.703_704insG TREX1 
gene mutation has been reported in Caucasian patients from The Netherlands, USA, Australia and Italy $1,3,10,11$. The participants of this study are from a village located in Mexico State, are Mexican Mestizos (parents and grandparents of Mexican origin) and belong to the same family. Thereby, we suggest that clinicians take a more thorough family history of patients with vascular disease.

Our clinical cases illustrate how this entity can be misdiagnosed as an isolated cerebrovascular ischemic event, or even as demyelinating diseases. Clinicians should be alerted of patients with an apparent demyelinating disease but with atypical MRI findings and include this entity in the differential diagnoses.

CRV was first reported in $1998^{12}$ in a family with an unusual history of brain tumors. Later, all the family members were found to carry a TREX 1 mutation ${ }^{1}$. The systemic variant of RVCL is characterized by the presence of pseudotumoral brain lesions with calcifications in the white matter, multiple sclerosis-like phenotypes, CNS vasculitis, and retinal disease $3,13,14$. Since this disorder can affect multiple organs and systems, patients show a heterogeneous clinical expression. Due to the emerging clinical picture with systemic manifestations, authors from a retrospective study of 78 RVCL patients from 11 unrelated families renamed the disease as "RVCL and systemic manifestations ${ }^{10 "}$.

Physicians should take into account some differences between RVCL, and multiple sclerosis: (i) The age of onset in multiple sclerosis has a peak of 20-40 years (mean age of 32 years) ${ }^{15}$, whereas RVCL symptoms commonly begin in the forties; (ii) although the involvement of the optic nerve is present in both conditions, in RVCL cases the pathophysiological phenomenon is of ischemic origin, and its recovery is little or null ${ }^{15}$; and (iii) overall, RVCL patients exhibit bilateral retinal signs ${ }^{16}$. Thus, both disorders differ in their natural history.

Regarding brain MRI studies, even when the white matter is affected in RVCL patients, these lesions have a mass effect and are accompanied by a sudden onset of neurologic symptoms. This scenario should alert physicians to consider RVCL within the differential diagnosis of demyelinating lesions related to multiple sclerosis ${ }^{17}$ or some glial neoplasia that definitely does not show this type of neurologic onset. Our clinical cases highlight that a brain biopsy helps to establish that the physiopathogenic mechanisms do not correspond to those typically described for demyelinating diseases, while helping to exclude primary brain neoplastic tumors ${ }^{18}$.

Those cases whose clinical presentation suggests a cerebral vascular disease (ischemic or hemorrhagic), meaning a sudden presentation of a neurologic deficit or the establishment of a subacute neurologic deficit with MRI findings suggestive of ischemia (in the first case) or demyelination (second case), may require a brain biopsy to reach a diagnosis. However, histologically, the biopsy may be misinterpreted as a neoplasm ${ }^{10,19}$. Hardy et al. ${ }^{11}$ reported two siblings with $\mathrm{RVCL}$; in one of them demyelinating, tumefactive lesions, cerebral vasculitis and neoplasia were considered as diagnostic possibilities, pointing to the frequent misdiagnosis of this entity. They explained that when these lesions are accompanied by smaller periventricular lesions, these may be mistaken for tumefactive demyelination. Regarding the radiological definition of "tumefactive lesion," it is not consistent throughout the literature and may refer to various combinations of size ( $>2 \mathrm{~cm}$ ), mass effect, edema, and/or atypical enhancement patterns. In our study, the smallest lesion identified by prebiopsy MRI was $0.5 \mathrm{~cm}$ in diameter; the lower and upper quartiles for the entire cohort were 2.5 and $6.0 \mathrm{~cm}$, respectively. In contrast, in a study of 168 cases, 13 patients presented lesion sizes of $<2.0 \mathrm{~cm}^{17}$.

A subset of RVCL patients develops systemic vascular involvement manifested by Raynaud's phenomenon or liver or renal dysfunction ${ }^{3}$. Our cases did not exhibit Raynaud's phenomenon, while hepatic alterations were observed in the three patients, and renal dysfunction was only found in patient 1 .

In our three clinical cases, two common characteristics were present: the sudden onset of neurological symptoms, and periventricular hyperintensities and pseudotumoral lesions as brain MRI findings (Table 1 and Fig. 1).

Brain manifestations have a prevalence of $81 \%$ including focal neurological deficits and migraine in more than $50 \%$ of the $\operatorname{cases}^{10}$. In our study, only patient 2 
had a history of migraine. The presence of positive anti-DNA auto-antibodies, as in Case 1, has been reported on two patients ${ }^{10}$. Taking into account the complete penetrance of TREX1 mutations ${ }^{1,4}$, the phenotypic variability in our three-related patients could be explained by a combination of other genetic and environmental factors.

There is only one report of a Taiwanese patient with a heterozygous TREX1 c.294dupA and a homozygous NOTCH3 c.1630C $>$ T (p.R544C) mutation with a modified phenotype of CADASIL $^{2}$. Thus, there is a possibility that genetic variants in genes encoding proteins interacting with TREXI or in other genes associated with small vessel's diseases of the brain can modify RVCL progression.

Regarding the different clinical progression between patient 2 and her two first-cousins, it might be attributed at least in part, to differences in lifestyle. Patient 2 showed a faster course and earlier age of onset of RVCL, with also a higher amount and time of cigarettes consumption than her cousins. RVCL is an autosomal dominant disorder with $100 \%$ of penetrance and unavoidable clinical manifestations ${ }^{1,4}$. The different clinical course in our three-related patients suggests that a healthy lifestyle and prevention of associated cerebrovascular disease risk factors might delay the age at onset and severity of this disorder. However, this needs to be proven in a longitudinal follow-up of the patients.

It is also important to note that the cases presented here received different medical treatment, raising the question of how best to manage RVCL patients. To date, there is no known treatment for the vasculopathy associated with this mutation. The most effective way to treat these tumor-like lesions is unclear. In the literature, no benefit has been observed from plasma exchange, IV immunoglobulins, or chronic immunosuppression $^{13}$. Di Francesco et al. reported a patient that was initially treated with immunosuppression without any substantial improvement although a transient stabilization of the clinical course was observed using low molecular heparin. They also included CPM in the treatment, but no additional information was provided ${ }^{3}$.

Case 1 was treated with rituximab and CPM and was apparently stable during the 24-month follow-up period after the clinical event. There is no documented data related to these treatment options; only one report suggests the use of natalizumab for $\mathrm{RVCL}^{11}$. It would be interesting to evaluate these therapeutic options for a longer period in these patients.

There is evidence to support the use of CPM for small vessel vasculitis ${ }^{20}$. Thus, it is reasonable to consider using CPM to treat patients with pseudotumoral CNS lesions. The cases presented here exhibited clinical heterogeneity, making diagnosis, and selection of a long-term treatment plan very difficult.

We identified 15 additional relatives who were premanifest TREX1 gene mutation carriers (Figs. 2 and S2). We will give them a strict clinical follow-up and support, including MRI studies to detect abnormalities in the pre-manifest phase of RVCL.

PD of RVCL was offered to all at-risk subjects, although only 11 relatives applied for it. As in other inherited diseases, few people wish to learn about their genetic status, mainly due to the lack of treatment options. The decision on whether to provide a diagnosis to pre-manifest TREX1 gene mutation carriers is complicated by medical, ethical, and legal issues.

Post-PD follow-up and support through a multidisciplinary team were offered to all of them. Furthermore, to include preventive lifestyle measures and minimize negative consequences of molecular testing, all participants were invited to attend at least one RVCLrelated health education session.

Each child born to an individual with RVCL has a 50\% chance of inheriting the pathogenic variant of TREX1, and this theoretical risk rises to $75 \%$ for the offspring of couples where both parents are carriers (Fig. 2) when including the possibility of homozygous alleles. The presence of consanguinity in two marriages raises new ethical dilemmas for geneticists. Interestingly, there have been reports of cases who were homozygous for TREX1 mutations related to different phenotypes: chilblains and cerebral vasculitis $^{21}$. Nevertheless, there have been no reports of RVCL cases, including a homozygous mutant TREX1 genotype.

Finally, the pre-manifest TREX1 gene mutation carriers (who will develop the disorder eventually) provide 
a model to study the early stages of the disease with directed interventions, and the opportunity to identify modifier factors of RVCL, as well.

\section{ACKNOWLEDGMENTS}

The authors would like to thank all participants in this study, with special thanks to Prof. Joanna C. Jen and Hafsa Mamsa, from the Neurology Department, UCLA School of Medicine for their involvement in this study.

\section{SUPPLEMENTARY DATA}

Supplementary data are available at Revista de Investigación Clínica online (www.clinicalandtranslationalinvestigation.com). These data are provided by the corresponding author and published online for the benefit of the reader. The contents of supplementary data are the sole responsibility of the authors.

\section{REFERENCES}

1. Richards A, van den Maagdenberg AM, Jen JC, et al. C-terminal truncations in human 3'-5' DNA exonuclease TREX1 cause autosomal dominant retinal vasculopathy with cerebral leukodystrophy. Nat Genet. 2007;39:1068-70.

2. Soong BW, Liao YC, Tu PH, et al. A homozygous NOTCH3 mutation p.R544C and a heterozygous TREX1 variant p.C99MfsX3 in a family with hereditary small vessel disease of the brain. J Chin Med Assoc. 2013;76:319-24.

3. Di Francesco JC, Novara F, Zuffardi O, et al. TREX1 C-terminal frameshift mutations in the systemic variant of retinal vasculopathy with cerebral leukodystrophy. Neurol Sci. 2015;36: 323-30

4. Kavanagh D, Spitzer D, Kothari PH, et al. New roles for the major human $3^{\prime}-5^{\prime}$ exonuclease TREX1 in human disease. Cell Cycle. 2008; 7:1718-25.
5. Federico A, Di Donato I, Bianchi S, et al. Hereditary cerebral small vessel diseases: a review. J Neurol Sci. 2012;322:25-30.

6. Lindahl T, Barnes DE, Yang YG, Robins P. Biochemical properties of mammalian TREX1 and its association with DNA replication and inherited inflammatory disease. Biochem Soc Trans. 2009; 37:535-8.

7. Orebaugh CD, Fye JM, Harvey S, et al. The TREXI C-terminal region controls cellular localization through ubiquitination. J Biol Chem. 2013;288:28881-92.

8. Alonso ME, Yescas P, Rasmussen A, et al. Homozygosity in Huntington's disease: new ethical dilemma caused by molecular diagnosis. Clin Genet. 2002;61:437-42.

9. Skirton $\mathrm{H}$, Goldsmith L, Jackson L, et al. Quality in genetic counselling for presymptomatic testing-clinical guidelines for practice across the range of genetic conditions. Eur JHum Genet. 2013;21:256-60.

10. Stam AH, Kothari $\mathrm{PH}$, Shaikh A, et al. Retinal vasculopathy with cerebral leukoencephalopathy and systemic manifestations. Brain. 2016;139:2909-22.

11. Hardy TA, Young S, Sy JS, et al. Tumefactive lesions in retinal vasculopathy with cerebral leucoencephalopathy and systemic manifestations (RVCL-S): a role for neuroinflammation? J Neurol Neurosurg Psychiatry. 2017 Aug 9 [Epub ahead of print].

12. Grand MG, Kaine J, Fulling K, et al. Cerebroretinal vasculopathy. A new hereditary syndrome. Ophthalmology. 1988;95:649-59.

13. Mateen FJ, Krecke K, Younge BR, et al. Evolution of a tumor-like lesion in cerebroretinal vasculopathy and TREX1 mutation. Neurology. 2010;75:1211-3.

14. Vodopivec I, Oakley DH, Perugino CA, et al. A 44-year-old man with eye, kidney, and brain dysfunction. Ann Neurol. 2016;79: 507-19.

15. Schuh E, Ertl-Wagner B, Lohse $P$, et al. Multiple sclerosis-like lesions and Type I interferon signature in a patient with RVCL. Neurol Neuroimmunol Neuroinflamm. 2015;2:e55.

16. Stüve O, Oksenberg J. Multiple Sclerosis Overview. In: Pagon RA Adam MP, Ardinger $\mathrm{HH}$, et al, editors. Gene Reviews ${ }^{\oplus}$. Seattle (WA): University of Washington, Seattle; 1993-2016. Available from: https://www.ncbi.nlm.nih.gov/pubmed/20301492 [Last updated on 2010 May 11]; [Last accessed on 2018 Jan 19].

17. Wallner-Blazek M, Rovira A, Fillipp M, et al. Atypical idiopathic inflammatory demyelinating lesions: prognostic implications and relation to multiple sclerosis. J Neurol. 2013;260:2016-22.

18. Ben-Nun A, Kaushansky N, Kawakami N, et al. From classic to spontaneous and humanized models of multiple sclerosis: impact on understanding pathogenesis and drug development. J Autoimmun. 2014;54:33-50.

19. Poser S, Lüer W, Bruhn H, et al. Acute demyelinating disease. Classification and non-invasive diagnosis. Acta Neurol Scand. $1992: 86: 579-85$

20. Hogan SL, Nachman PH, Wilkman AS, Jennette JC, Falk RJ. Prognostic markers in patients with antineutrophil cytoplasmic autoantibody-associated microscopic polyangiitis and glomerulonephritis. J Am Soc Nephrol. 1996;7:23-32.

21. Kisla Ekinci RM, Balci S, Bisgin A, Altintas DU, Yilmaz M. A homozygote TREX1 mutation in two siblings with different phenotypes: chilblains and cerebral vasculitis. Eur J Med Genet. 2017; 60:690-4. 